\title{
$\mathrm{CG}^{+} \mathrm{HR}$
}

\section{Africa's Voices: Using mobile phones and radio to foster mediated public discussion and to gather public opinions in Africa}

\section{Claudia Abreu Lopes}

Research Associate, Centre of Governance and Human Rights, University of Cambridge

\section{Sharath Srinivasan}

Director, Centre of Governance and Human Rights, University of Cambridge 


\section{Centre of Governance and Human Rights Working Papers}

The Centre of Governance and Human Rights (CGHR) draws together researchers, practitioners and policymakers from the University of Cambridge and far beyond to think critically and innovatively about pressing governance and human rights issues throughout the world, with a special focus on Africa. The Centre aims to be a world-class interdisciplinary hub for fresh thinking, collaborative research and improving practice.

The CGHR Working Papers Series is a collection of papers, largely peer-reviewed, focussed on cross-disciplinary research on issues of governance and human rights. The series includes papers presented at the CGHR Research Group and occasional papers written by CGHR Associates related to the Centre's research projects. It also welcomes papers from further afield on topics related to the CGHR research agenda.

Series Editors: Sharath Srinivasan \& Thomas Probert

Publisher: Centre of Governance and Human Rights, University of Cambridge

Contact: cghr@polis.cam.ac.uk, (+44) (0)1223 767257 


\begin{abstract}
This paper presents the findings from a one-year applied research pilot project, Africa's Voices, run by the University of Cambridge's Centre of Governance and Human Rights (CGHR). Africa's Voices developed out of CGHR's wider research programme on politics, ICTs and interactive media in Africa. That research analyses how audiences interact with radio stations through mobile phones; how different actors including audiences, radio journalists, and governance actors (state officials, but also others such as community leaders and aid actors) perceive the importance of these interactions; and what the practical implications are for public discussion of political and social issues and for governance processes that shape access to and the quality of public goods. With Africa's Voices, the CGHR research team piloted a programme format with local radio stations in eight sub-Saharan African countries with the objective of practically assessing the potential for deploying interactive radio to gather and comparatively analyse opinions of harder to reach sub-Saharan African populations. Besides evaluating optimal modes of working with smaller and more rural radio stations, the research has focused on patterns of audience participation in different formats of mediated public discussions and on the efficacy of different approaches to defining, gathering and measuring public opinion. This paper presents the results of the pilot and discusses them with respect to the abovementioned objectives. The paper also discusses some of the methodological and ethical challenges of using the affordances of ICT and interactive media that make them suitable for gathering and researching citizens' opinion in Africa.
\end{abstract}

\title{
1. Introduction
}

The media landscape in Africa is being transformed by the cross-fertilization of old media (radio and television) with new information and communication technologies (mobile phones, computers and internet). Hybrid and convergent media infrastructures are enabling journalists as well as audiences to generate content in new ways, for listeners/viewers to participate in talk shows and audience polls and for a range of third party actors to 'intervene' to create interactive programmes on specific issues. More than previously was the case, media institutions have come to be seen as "participatory organizations" (Willems, 2013) as private opinions are communicated and amplified by the media reaching other citizens, governments, companies, and international actors.

Despite growth in television and, from a low base, rapidly rising internet penetration and use of social media, radio remains the dominant media channel across the continent in part due to its geographic reach, the low-cost of equipment and the versatility to operate with different sources of energy. Following media liberalization in many countries in the 1990s, the African radioscape has progressively become richer and more diverse. Local language broadcast, commercial, community and religious operators, and internet-based broadcasting have served to further extend radio's importance. It is a medium that also resonates with and augments dominant oral cultural practices in many African societies. On the heels of radio's efflorescence, mobile phone penetration has rapidly risen across the African continent, which in 2012 was the fastest growing and second biggest mobile market in the world with roughly 750 million handsets for its one billion people (ITU, 2013).

Interactive radio thus arises out of a relatively recent revolution in media and communications, with much work yet to be done to assess its potential for expanding African public spheres. New public spaces of discussion are being created with diverse forms, but are uniquely characterized by their spatial reach, communicative immediacy and their higher density of interactions. Changes in the production of popular culture, the generation of news, the social life of meeting and greeting are some of these manifestations alongside debates on social issues, politics and development. The latter exhibits interesting potentialities, such as the possibility of exposing facts, ideas and beliefs to public scrutiny and allowing for them to be reinforced or contested by citizens holding similar or different worldviews. 
By challenging and enriching existing collective representations, radio-mediated public discussions are polyvalent in possible social effects: such social interconnectedness might strengthen social cohesion and yet if fragmented and mediated in certain ways it may deepen existing social cleavages. As new ways of thinking are voiced and incorporated, existing practices and social norms are challenged, leading to social change processes that, however, gradual, are novel and distinct. At the same time, ordinary citizens may be empowered with new tools to get to know and exercise their rights and to voice their demands. By encouraging audiences to express their opinions, interactive media can be perceived as a tool for expanding the freedom of individuals (Sen, 1999) and in particular their social or political "capability" (Srinivasan, 2007), lending credibility to the label of new ICT as "technologies of freedom" (Willems, 2013). Nevertheless, it is by no means clear whether or not expansion of interactive media leads on balance to more inclusive or more democratic practices, more transparent governance or more just and efficient delivery of public goods. What is clear, and of interest here, is how these new mediated 'public' spaces might enable different expressions of public opinion.

This paper presents the findings from a one-year applied research pilot project, Africa's Voices, run by the University of Cambridge's Centre of Governance and Human Rights (CGHR). Africa's Voices developed out of CGHR's wider research programme on politics, ICTs and interactive media in Africa. That research analyses how audiences interact with radio stations through mobile phones; how different actors including audiences, radio journalists, and governance actors (state officials, but also others such as community leaders and aid actors) perceive the importance of these interactions; and what the practical implications are for public discussion of political and social issues and for governance processes that shape access to and the quality of public goods. Qualitative case-study research (using ethnographic work in studios, analysis of interactive programmes, audience surveys and key informant interviews) has been conducted in Kenya, Uganda and Zambia, enabling a comparative basis for exploring how different historical, social, cultural and political factors influence the ability of new ICTs to affect governance processes.

With Africa's Voices, the CGHR research team piloted a programme format with local radio stations in eight sub-Saharan African countries with the objective of practically assessing the potential for deploying interactive radio to gather and comparatively analyse opinions of harder to reach sub-Saharan African populations. Besides evaluating optimal modes of working with smaller and more rural radio stations, the research has focused on patterns of audience participation in different formats of mediated public discussions and on the efficacy of different approaches to defining, gathering and measuring public opinion. This paper presents the results of the pilot and discusses them with respect to the abovementioned objectives. The paper also discusses some of the methodological and ethical challenges of using affordances of ICT and interactive media that make them suitable for gathering and researching citizens' opinion in Africa.

\section{Debates on ICT, Interactive Radio and African Participatory Governance}

CGHR's research programme, including the Africa's Voices component, intervenes at the intersection of three distinct but related literatures. The first of these, a mostly policy-oriented 'ICT for Governance' literature considers how new ICT can be used to enhance 'demand-side' governance and accountability through a range of interventions, such as mobile phone enabled citizen scorecards, election information campaigns targeting citizens, new ICT-based channels for interest articulation vis-à-vis elected representatives and authorities, etc. (for example, see Bertot et al, 2010). Many of these have become the focus of randomized control trials and similar experimental studies. A large donor effort branded 'Making All Voices Count' suggests that trends of open government, open data, big data and new ICT are seen as central to future directions in 
pro-poor development and governance programming. In the words of an official from one sponsor agency, the Swedish International Development Agency: "In government, ICT may increase accountability and transparency, and counter corruption through more efficient administration and increased flows of information. This may also strengthen good governance and improve interaction between government and citizens" (SIDA, 2009).

Yet at the same time, the wider 'ICT4D' research community has come to share long-standing concerns that a greater focus be placed on contextual appropriateness, on technologies in use, on local application and innovation and on scaling up the existing successes (Heeks, 2008). An interest in technological applications that better interact with the political and social forces operating in different contexts has developed in parallel with the attention paid by scholars to how domestic social and political actors appropriate, re-shape and adapt technologies to fit in pre-existing networks and in so doing contribute to their partial re-configuration (Gagliardone, 2010; Morawczynski, 2009). Technologies are multi-purpose artefacts whose social meanings change depending on places of use and origin through sustained dialogical encounters. With innovation hubs springing up across Africa on the back of successes such as the Kenyan developed crowdmapping platform Ushahidi and mobile money system M-PESA, or the South African social media phenomenon MXit, the trend heeds a long-standing call for new tools which build on local knowledge and habits and allow users to progressively shape and master innovation, rather than be forced into new programmes from above. To take a human development approach, the transformative potential of ICT needs to be studied not in relation to outcomes defined a-priori but with the capacity for individuals to be empowered to attain outcomes of their own choosing (Kleine, 2010). Just as with technological artefacts and their uses, guiding notions such as 'participation' and 'voice' must also be reappraised in ways that overturn generic templates for positive social change cast out of an imported and over-idealized Western mould.

A second literature on African media, including 'media for development' praxis scholarship and studies of audience participation in radio programmes, is also at times circumspect with regards to the dynamics of change occurring. There is little doubt that since the 1990s, African mediascapes have been transformed by a growing liberalization of the media in many countries (Nyamnjoh, 2005; Spitulnik, 2002; Wasserman, 2011). New ICT proliferation has further marked this period of change (Willems, 2013; Mare, 2013; Myers, 2008; Moyo, 2013; Gratz, 2013). As Chiumbu (2013, p.242) has noted:

Although the radio medium has always encouraged deliberation and participation through phone-in programs and studio audiences, [...] ICTs have changed the nature of this participation by expanding the discursive spaces and drawing audiences closer to production and the station's institutional processes.

Mabweazara argues that this has allowed listeners to 'speak back' to radio stations content as well as to participate actively in the generation of content (see Mare, 2013, p.31). The use of new ICT may be revitalising African radios by opening participative venues and their potential for citizens' involvement in public debate, but interactivity on African radios remains embedded within existing social and political hierarchies and practices, thus whether it amplifies or adjusts existing patterns of participation and identification will vary with context, event and time (Brisset-Foucault, 2009; Schulz, 1999; Vokes, 2007; Willems, 2013).

Considerable interest continues to be expressed in how interactive radio discussion, perhaps motivated by 'civic journalism,' might be contributing to the development of African 'public spheres' that have the potential to be modes of societal integration and coordination of human life distinct from state power, traditional authority and market economies. Yet the political 
economy of radio stations is such that commercial priorities of the stations as well as third-party considerations - including how political figures shape and leverage the radio station within their strategizing and the imperatives of governmental and development actor sponsors of radio programmes - play a considerable role in the kinds of 'participatory spaces' created (and foreclosed) through audience interactivity (Willems, 2013). There is thus a tension between the potential for more inclusive and robust public discussion through radio-mediated formats and what Habermas (1991, p.187-8), when addressing the ills of twentieth century corporate mass media, referred to as a "power penetrated public sphere ... altered to the influx of private interests..." "Radio stations," he noted, "have turned the staging of panel discussions into a flourishing secondary business [where] discussion seems to be carefully cultivated [and] assumes the form of a consumer item" (Habermas, 1991, p.164).

Finally, there is growing interest in the role of 'public opinion' in African politics and current affairs (Bratton, Mattes \& Gyimah-Boadi, 2005; Wolf, 2009), and this includes some attention paid to the rising role of the media in gathering and representing public opinion. A drive for 'accountability through public opinion' contends that "governments will only be accountable if there are incentives for them to do so-and only an active and data and media literate public will change the incentives of government officials to make them responsive to citizens' demands" (Odugbemi \& Lee, 2011). This begs the question of who makes 'public opinion', what types of 'public opinion' matter, and how? What if there are multiple publics? How useful are ICT and media in enhancing public opinion gathering tools compared to other channels, spheres and institutions? How do 'traditional' political actors and opinion makers use ICT and interactive broadcast media?

There is a risk of defining 'public opinion' reductively because it is efficacious to do so. Large-n and in-person survey processes have strengths but also weaknesses, notably in how they 'aggregate' individual answers to closed questions to generate an artificial gauge of 'public opinion' at an abstract moment in time. The uniqueness and context of opinions are often lost in large-scale surveys, as well as the dynamic, socially constituted nature of shared ideas, yet alternatives, such as focus group discussions and ethnographic studies are highly circumscribed in terms of place and population.

A shift of focus to more interactive and social spaces of public opinion formation suggests reflection from the perspective of the deliberative democracy school of thought. Where public opinion is generated within radio programme formats, the radio discussion might add deliberation to aggregation, while also introducing new forms of bias and contingency. There may be potential to draw upon what James Fishkin's $(1991,2009)$ conception of "deliberative polls", premised on the normative claim that only opinions which are gathered amongst a representative group after giving out information and allowing for a deliberation are legitimate and efficacious. Whether or not the mediated, punctuated and somewhat chaotic nature of interactive radio shows can adequately embody a 'discourse ethic' is highly questionable. The determinants of 'good' or 'credible' deliberation are subject to debate and often hard to measure or validate, such that a space populated by different voices and opinions might not be very deliberative at all.

Deliberation theories usefully place emphasis on the importance of voices expressed in public discussion and recognition of such voices in a healthy democratic polity, but in the ideal they demand too much of rationality in procedure (discussion) and consensus in outcome (agreement). Alternative approaches, such as that of Hannah Arendt $(1963,1971)$, privilege the many-sidedness of human affairs and the inevitability of struggle, and reinforce the importance of a plurality of opinions in public spaces as being at the heart of healthy polities. The value of voice as a process of organizing human experience through interconnected narratives is embedded in social and political frameworks where voices can be heard and are effective to change practices (Couldry, 
2010). In such contexts, voices are privileged access points to individual and shared significations that are a vehicle for social change. The access point is also a human moment of recognition. $A$ different idea of public opinion is employed when we value voice in this way. Here, the role of interactive media is especially significant in heralding new possibilities for the place of public opinion in processes of governance and development on the African continent.

Africa's Voices, then, allows CGHR researchers to explore tensions between, on the one hand, the interventional desire for scalability, replicability, parsimony and efficiency in the convergent use of ICT and radio to promote engaged citizen participation in governance and, on the other hand, the need to under-determine the manner of adoption of technology into station and audience practices so as to leave space for indigenization of technology-based interactive practices and the generation of contextually specific meaning (whether the content of 'voice' or the value attached to the expression and reception of that voice).

\section{Africa's Voices Pilot Study: Developing a Methodology for Public Opinion Gathering Using Interactive Radio}

Africa's Voices capitalizes on the pervasiveness of radio and mobile phones across the continent to gather and analyse the views of populations that are usually under-represented in public discussion, and that are time and resource intensive to access with traditional methodologies such as face-to-face surveys, focus groups and ethnographical studies. From an applied side, the project aims to help radio stations promote interactive discussions on air and in audience communities, motivated by evidence of what people in the same or other communities think about topical issues. One wider objective here is the efficient, timely and credible generation of knowledge of public opinion and social realities that can be used by governments and development actors and by the wider research community. Researchers building knowledge in such fields as African politics, public health, international development, social anthropology, and social psychology might also deploy the methodologies developed. In future scaled-up scenarios, information collected by Africa's Voices could be aggregated into public opinion indicators used, for example, by governments as feedback for policies and reforms, supporting more participatory and accountable governance processes, or by development organizations to monitor and evaluate their programmes locally. By promoting discussion within and across communities, Africa's Voices facilitates encounters between contrasting worldviews, which might challenge existing social representations (Moscovici, 1961) which are collective beliefs that are socially created, transformed and shared through everyday communication. The perception of a 'collective voice' distinct from other voices may also contribute to the strengthening a sense of social identity (Tajfel and Turner, 1986) among audiences fostering intergroup dynamics conducive to collective decision-making and to certain forms of mobilization.

From an academic perspective, the Africa's Voices research pilot seeks to build on the understanding of the nature of public opinion that the combined use of mobile phones and radio promotes through an intervention that expands the quality and depth of mediated public sphere discussions, and sustains audience consultation and engagement in media programming. In order to do this, the research seeks to understand how technology that allows bi-directional participatory communication is deployed by audiences and by radio stations. Beyond the pilot phase, CGHR is giving greater focus to the nature and significance of citizens' 'voice' and selfperceptions of socio-political agency generated through audience interactivity which may spread to other forms of political participation.

\section{Pilot Objectives}


During its one-year pilot, CGHR tested the characteristics of effective methodologies for stimulating interactive radio debate and collecting opinions from radio audiences using short message service (SMS). At the same time, the project sought to understand how radio stations incorporate new ICT such as FrontlineSMS and FreedomFone: open source software for the collecting and management of SMS from a computer with limited internet access, in order to create new spaces for audience participation and new configurations for interactive radio. The evidence was used to reflect on how audiences and stations engage with poll-oriented modes of public opinion gathering in a variety of social and cultural contexts.

After the pilot stage which was more focused on the operational method and on establishing the viability of an applied intervention of this kind, the project shifted to substantive enquiries. The research team seeks to understand the contours of public opinion conveyed by public discussions mediated by ICT (radio, online platforms) and to develop analytical tools that aggregate individual opinions into meaningful collective representations, taking into account the knowledge of the socio-cognitive processes and the socio-cultural contexts that enable their emergence. The project also investigates how these new spaces of discussion may support public goods provision within local governance settings, for example, through the diffusion of knowledge and information, as well as the significance of more involvement of citizens in community life, engagement in collective action and reinforcement of accountability and transparency practices.

The pilot was guided by four research questions:

(1) What is the optimal socio-technological workflow for an intervention such as Africa's Voices, including its capacity to accommodate specificities of particular stations?

(2) What kinds of audience participation do an intervention such as Africa's Voices enable, what kinds does it limit, and which are given preference by audiences and stations?

(3) Does Africa's Voices help to create new and more inclusive spaces for interaction between radio stations and listeners and/or does it reinforce status quo bias in debate and discussion?

(4) What is the perceived significance of Africa's Voices for the radio stations and for the community? What credible models can be identified for enabling interventions such as Africa's Voices to enhance individual and collective engagement in socio-political affairs and citizen-led governance?

\section{General Methodology}

During the Africa's Voices' pilot, CGHR implemented a quasi-experiment that manipulates key factors relating to the procedure of collecting opinions from radio audiences through SMS. The effect of these manipulations was assessed in three dimensions: (1) level and quality of audience participation; (2) adoption of new technologies in radio stations' routine; (3) empowerment of journalists and communities by engaging in interactive radio. The research combined diverse sources of information to derive process and outcome indicators: web-based questionnaires and telephonic interviews with journalists; participant observation in radio stations; focus-group discussions with radio listening groups; and quantitative analysis of datasets of SMS.

Every month, the radio stations involved in the pilot asked their listeners questions, to be answered via SMS to a number linked to one computer in the station (connected to a modem). The topic and content of the questions were suggested by and discussed with all the stations in order to adjust them to local realities and relevance. The final questions were designed by the Africa's Voices team in Cambridge and tested in two radio stations with high levels of commitment to the project and strong technological capacity before being rolled out more widely. Africa's 
Voices rounds of questions were implemented in a staggered and incremental manner, so that the feedback from these lead stations could be incorporated into the questions to be asked in the other stations.

Africa's Voices questions were asked during a weekly programme for two consecutive weeks. The programme was devoted to Africa's Voices to avoid contamination by other topics or contents. The Africa's Voices question was introduced through a script translated into local languages to ensure that the questions were framed similarly in all stations. The script consisted of a summary of basic information about the topic, with country references and arguments of different sides of the issue, followed by the question and answer instructions using a round-specific keyword. The script also contained a brief explanation of the project methodology and relevant stakeholders (including University of Cambridge). Listeners were also advised not to send personal information with the answer to ensure anonymity of the data stored in the radio stations and were warned about the possible consequences in terms of disclosure of personal data linked to mobile numbers. The SMS were collected in the station's computers using FrontlineSMS or FreedomFone, both freely available software for SMS aggregation and management. In some stations, the question was also asked throughout two weeks in a jingle containing the same information as the script. In either case, the answers received at the station were read out during the Africa's Voices' programme to encourage participation and energize the on-air debate. After the question was asked over two weeks, the SMS related to the Africa's Voices were filtered by keyword in the radio stations and sent by email or Dropbox to CGHR at the University of Cambridge for qualitative and quantitative analysis. For each round of questions, the CGHR team produced a brief report that was sent to the stations with customized information (poll results for the station and comparison with other stations) and the results uploaded in the Africa's Voices website (percentages, themes and socio-demographics) as well as the SMS translated into English with personal references omitted.

\section{The Pilot Stations}

After a pre-trial set-up phase, the project started in September 2012 with ten stations in Kenya (Radio NamLolwe, Radio SautiFM and Radio Pamoja), Uganda (Radio BudduFM and Radio BetterFM), Malawi (Radio Mudzi Wathu), Zambia (Radio BreezeFM), Ghana (Radio RiteFM), Sierra Leone (Radio Gbafth) and Mozambique (Radio Corredor da Beira). All these stations have a local focus and play a role in societal and political life in their communities. They encompass a variety of styles of management, ownership and technical capabilities and combine to different degrees a community-oriented radio model with a more commercial approach.

In addition to the head of the station evidencing involvement in the project, the sine qua non conditions for radio stations to be included were having: (1) relatively stable power; (2) a computer with a reasonably reliable internet connection (through a modem, broadband or wi-fi); (3) basic ICT knowledge of the key person(s) responsible for implementing the project in the station; (4) a community focus; (5) a broad focus with different programmes addressing governance and development topics; (6) adequate management and staffing. Some of these stations were chosen because they were piloting the software FrontlineSMS:Radio. Other stations had voluntarily contacted the CGHR expressing interest to be part of the project. None of the Africa's Voices programmes were sponsored by third parties, so the content and format could be decided between the CGHR team and the head of programs and/or presenters.

After initial contact, the CGHR assessed the suitability of the stations for the pilot in terms of the interest of managers and journalists, capacity (power, equipment, IT knowledge and internet connection) and complementariness of existing programming. A web questionnaire was sent to all 
stations to identify the ICT equipment owned, energy and internet access and other characteristics of the stations (e.g., antenna reach, number of employees, etc.). If the station met the criteria, one employee was identified by the management team to take responsibility for the project in that station, usually a presenter or head of programmes who was ICT literate. The Africa's Voices representative in the station had to be motivated to implement and manage the project in the station and available to communicate regularly with the Africa's Voices team in Cambridge.

Six of these stations successfully implemented the first round of questions (baseline) but four others failed to do so due to lack of technological capacity (malfunctioning of ICT equipment) and/or disrupted communications (e.g., slow response time). Four stations were suspended from this initial stage of the project (in Kenya, Pamoja and SautiFM; in Uganda, BetterFM; and in Mozambique, Radio Corredor da Beira). Based on advice given by FreedomFone and contacts in the field, three new stations were added successfully after the first round: in Malawi, Radio Nkhotakota; in Tanzania, Radio Ulanga; and in Mozambique, Radio Comunitaria da Gorongosa. The pilot thus proceeded with nine participating stations in eight countries.

\section{The Quasi-Experiment}

The pilot was designed as a quasi-experiment (Shadish, Cook \& Campbell, 2002) - with manipulation of independent variables and no random assignment of cases to conditions - with the aim of evaluating the impact of variations in the procedure of asking and gathering questions from radio audiences, at different times, with reference to a baseline. A repeated measures design considered different time points defined by rounds of questions asked to radio audiences with an interval of approximately one month, following the same sequence. The stations were allowed to adjust some procedures to match their own practices (e.g., using a jingle to ask the question) but the core methodology of the quasi-experiment was invariant across all of the nine stations.

As an applied research project with a real effect on the participants involved (radio stations audiences, and communities), the research design did not incorporate a control group as it would have meant preventing some stations/communities from benefiting from the anticipated positive effects of incremental improvements in the poll methodology. As an alternative, we set up a baseline (round 1) by using the same poll methodology in all stations and compared the audience participation in successive rounds of questions with the baseline results.

A major weakness of this type of design is the "history effect" (Cook and Campbell, 1979; Shadish, Cook \& Campbell, 2002), which leads to confounding if a change in the dependent variable relates to a distinctive historical event and be wrongly attributed to the manipulation of an independent variable. As the quasi-experiment was run in several countries, it is very unlikely that an external event could have had a comparable impact in all countries.

Nevertheless, the quasi-experimental design has some flaws resulting from our "incorporating learning as we go" approach. When a specific procedure (e.g., using-mixed format answers) was found to have positive effects compared with the baseline, this procedure was adopted in all of the stations in subsequent rounds. It was not therefore possible to consider a quasi-experimental design with the full combination of levels of procedural variables, as ineffective procedures were not incorporated from subsequent rounds. As such, the quasi-experiment has a molar approach: testing different procedures through rounds of questions, combining particular poll features (e.g. binary question with mixed format, non-word keyword, local language and informed consent). Therefore, the independent variable of the quasi-experiment is the poll methodology. The dependent variables are quantifications of engagement of audiences and stations and, of characteristics of the answers received from the audiences. 
As in different rounds different questions were asked, the effects of the poll methodology may have been confounded by the specific content of the questions. It is possible that the "success" of one methodology was more dependent on the content of the question rather than its format. In addition, listeners interested in different topics may have different characteristics that influence the way they answered the poll. To reduce this confounding effect, we asked questions related to the same topics (development and governance) and of general interest to all types of audiences. Using this framework, the particular questions were designed collaboratively between the Africa's Voices team, radio presenters and local collaborators.

The validity of a repeated measures design may also be undermined by the effects of practice and fatigue (Cook and Campbell, 1979; Shadish, Cook \& Campbell, 2002). In the context of this research, the audiences may have become more responsive to the polls over time as their interest increased, more familiar with answering formats, or more confident sending SMS to radio stations. Alternatively, the audiences may have lost interest in the polls over repeated questions, resulting in a drop in participation over time. Considering the wide time gap between different rounds (one to two months), and the fact that the groups of respondents vary from round to round, it is unlikely that these potential effects may offer an alternative interpretation of the results.

\section{Variables and Procedure}

The baseline of the quasi-experiment was set in round 1 for all the stations. Five factors were manipulated: (1) type of question; (2) format of answer; (3) language of the question; (4) type of keyword; (5) anonymity request (cf. Table 1). The impact of the variations of these factors was assessed by considering the characteristics of the answers (recorded in databases of SMS) and of feedback received through an online questionnaire that was sent to the Africa's Voices representatives in the stations at the end of each round.

The dependent variables were: (1) the engagement of audiences with the Africa's Voices polls (number of stations involved in the round and number of messages received); (2) the characteristics of messages received (format of SMS, presence of justifications, language of the answer, use of keyword, and personal information included); (3) and objective indicators of stations' performance (average response time across all stations between sending the final script to the stations and asking the question to the audiences, and sending the SMS spreadsheet to (GHR).

Seven questions were asked in the Africa's Voices pilot:

(1) Pre-pilot round: "What is the main threat to food security in your area? A: climate change; B: land access; C: market access; D: food prices"

(2) Round 1: "Should our government ban the use of plastic bags? Yes or No?"

(3) Round 2: "Which one of these two statements do you agree with? 1: African presidents should be limited by their country's constitution to serving a maximum of two terms in the office OR 2: Africans should be able to democratically elect a president to serve for more than two terms"

(4) Round 3: "What is the minimum number of years that children should be required by the government to attend school?"

(5) Round 4: "Which one of these diseases do you fear more? AIDS or malaria? Why?"

(6) Round 5: "Do you think a 16-year old girl [local fictional name] should interrupt her education to get married to help the family of should continue her studies to seek a better future for her and her family? Why?" 
(7) Round 6: "Do you feel safe walking alone in your area after dark? Yes or No? Why?"

Table 1: Quasi-experiment for testing Africa's Voices methodology

\begin{tabular}{|c|c|c|c|c|c|c|c|}
\hline & Pre-pilot & Round 1 & Round 2 & Round 3 & Round 4 & Round 5 & Round 6 \\
\hline $\begin{array}{l}\text { Type of } \\
\text { question }\end{array}$ & $\begin{array}{l}\text { Multiple } \\
\text { choice }\end{array}$ & $\begin{array}{l}\text { Binary } \\
\text { Yes/No }\end{array}$ & $\begin{array}{l}\text { Binary } \\
\text { (two sentences) }\end{array}$ & $\begin{array}{l}\text { Numerical } \\
\text { answer }\end{array}$ & $\begin{array}{l}\text { Binary } \\
\text { (AIDS/Malaria) }\end{array}$ & $\begin{array}{l}\text { Binary } \\
\text { vignette }\end{array}$ & $\begin{array}{l}\text { Binary } \\
\text { Yes/No }\end{array}$ \\
\hline $\begin{array}{l}\text { Format of } \\
\text { answer }\end{array}$ & Closed & Closed & Closed & $\begin{array}{l}\text { Open } \\
\text { numerical }\end{array}$ & Mixed & Mixed & Mixed \\
\hline Language & English & English & Local & Local & Local & Local & Local \\
\hline Keyword & $\begin{array}{l}\text { Word } \\
\text { Food }\end{array}$ & $\begin{array}{l}\text { Word } \\
\text { Bag(s) }\end{array}$ & $\begin{array}{l}\text { Non-word } \\
\text { AV }\end{array}$ & $\begin{array}{l}\text { Non-word } \\
\text { AA }\end{array}$ & $\begin{array}{l}\text { Word } \\
\text { Fear }\end{array}$ & $\begin{array}{l}\text { Word } \\
\text { Marry }\end{array}$ & $\begin{array}{l}\text { Word } \\
\text { Safe }\end{array}$ \\
\hline $\begin{array}{l}\text { Anonymity } \\
\text { request }\end{array}$ & Strong & Strong & $\begin{array}{l}\text { Very } \\
\text { strong }\end{array}$ & Very strong & $\begin{array}{l}\text { Informed } \\
\text { consent }\end{array}$ & $\begin{array}{l}\text { Informed } \\
\text { consent }\end{array}$ & $\begin{array}{l}\text { Informed } \\
\text { consent }\end{array}$ \\
\hline
\end{tabular}

Type of question. Rounds 2 to 6 were compared to the baseline (round 1) and evaluated against each other in terms of the characteristics of the messages in addition to engagement of the audiences and the stations. After an initial unsuccessful attempt to ask a question with four alternatives answers in a pre-pilot stage, the options were kept as simple as possible with binary options such as yes/no (round 1 and 6), selecting one sentence out of two (round 2) or selecting one of two words (round 4). Round 3 sought a numerical answer and round 5 a binary answer in order to decide the ending of a story vignette.

Some studies have showed (see Sudman, Bradburn \& Schwarz, 1996) that primacy and recency effects in answering questions (over focus on the first or last option, respectively) depend on the mode in which they are presented. When presented in oral format (vs. written), the recency effects are more pronounced and the last option presented is given more attention and thought. This issue was taken into account in round 2 ("African presidents should be limited by their country's constitution to serving a maximum of two terms in the office or Africans should be able to democratically elect a president to serve for more than two terms") as the more complex and less intuitive option was presented at the end (involving the ideas of democratic elections and limited terms). The binary choice questions are all diametrically opposed, as agreeing with one implies necessarily disagreeing with the other.

Format of answer. Regardless of the format of the questions, the format of the answer can be open-, close-ended or mixed. Closed answers only offer the possibility of selecting a predefined set of answers while mixed and open formats allow a more diverse and unrestricted range of opinions. The first two rounds allowed only closed-ended answers with a choice between yes and no. The subsequent rounds allowed open answers, whether in a numerical format (round 3) or a mixed textual format (round 4 to 6), combining binary options with justification of the choice. The virtues of close-ended answers with standardized options relate to the ease of aggregation and possibility of rigorous and powerful statistical analysis. But they pose limits on individual expression, creating artificial and often simplistic opinions disengaged from the original context or knowledge. The standardized answer formats also pose a threat to representativity by excluding 
those citizens with poor survey literacy or technological skills. By favouring mixed format polls, Africa's Voices recognizes the benefits of using free textual forms to promote inclusivity and diversity. But as an opinion polling innovation, the project explores strategies of aggregating answers, calling for more standardized forms of opinion gathering.

Language and wording of the questions. The first two questions were asked in English and the subsequent questions were asked in the main languages in which the stations broadcast (e.g., Portuguese, Kiswahili, Chichewa, Chinyanja, Luo, Luganda and Krio). However, the keyword was always the same across all stations (mainly in English) due to the requirements of the aggregation software. For close-ended answers, the codification process ignores the language in which the question was asked. When standardized answers were accompanied with textual information in local languages, the analysis of SMS is more laborious, time consuming and open to multiple interpretations. On the one hand, asking questions in English may prompt answers also in English, making them easier to handle, but on the other hand, the audience participation, the popularity of programmes, and reach of the polls may all be negatively affected. The language used can also influence the content of answers. A study by McIntosh (2010), comparing the use of English vs. Kiswahili in Kenya, suggested that sending SMS in English tends to be associated with a 'fantasized person' of infinite choice and freedom, resembling the compressed and stylized character of text messages, as opposed to messages written in local languages that allow for articulating elements of moral ethnicity and social obligations. Due to the more genuine character of answers given in local languages, the project tended to welcome messages in any language. The translations of SMS into English were carried out by a group of volunteers, all students at the University of Cambridge, who were also native speakers of the local languages.

Use of keywords. The SMS must contain a keyword so that the stations can filter the messages related to Africa's Voices and send the resulting spreadsheet to the CGHR team in Cambridge. This procedure seeks protect the privacy of the stations and presenters by ensuring that the team in Cambridge reads only the SMS related to Africa's Voices. The round-specific keywords were either short words associated with the question (e.g., BAG or FEAR, MARRY, SAFE) or acronym/codes with two letters (e.g., AV, AA). The keyword was introduced in the script that the presenters were instructed to read aloud when asking the question on air.

Anonymity request. Audiences were always asked by the presenters to send anonymous messages. However, the level of stress placed in the request varied for different rounds, using strong, very strong and weak formulations in the questions script:

(a) Strong: "The messages should be anonymous so please do not include any personal information on it". (round 1)

(b) Very strong: "The messages should be anonymous so do not include any personal information. If you write your name in the message, your phone number and answer will be linked to your name". (rounds 2 and 3 )

(c) Weak (informed consent): “The messages should be anonymous so you don't need to include any personal information on it. If you decide to write your name, your phone number and answer will be linked". (rounds 4 to 6 )

The responsibility of stations to read this script is a weakness in the methodology, as the CGHR team does not often have recordings of the programme to consult, relying on the information provided by the presenters. Importantly, the anonymity of answers does not prevent senders from being identified by their mobile phones numbers, necessarily attached to the SMS. Radio stations unavoidably have this data to hand. 
From a research perspective, given the possibility that personal information is included in the SMS, the Africa's Voices team sought to comply with ethical research standards for handling confidential information by anonymising the answers that appear in the website (deleting personal information), masking mobile numbers in the SMS datasets, and limiting the access to the datasets using a password and a secure internet connection from the University. Again, however, the raw data files sent to Cambridge through email and Dropbox did include mobile phone numbers and personal references. These vulnerabilities and ethical concerns are thus an active focus of the redesign that will be done in the post-pilot phase of the project.

\section{Results}

The manipulations of the independent variables through a quasi-experimental design were assessed using quantitative evidence and key informant interviews with radio station managers and presenters (cf. Table 2). The results are presented for each independent variable, although the experiment adopted a more molar approach in which each round of questions combines different levels of the independent variables (cf. Table 1). The evidence was analysed by comparing the outcomes of different rounds, or groups of rounds, which differ from each other in terms of the level of the independent variable manipulated.

\section{Table 2: Results of quasi-experiment for Africa's Voices methodology}

\begin{tabular}{|c|c|c|c|c|c|c|c|c|c|}
\hline & \multicolumn{2}{|c|}{$\begin{array}{c}\text { Engagement of } \\
\text { stations and } \\
\text { audiences }\end{array}$} & \multicolumn{5}{|c|}{ Characteristics of the messages (percentages) } & \multicolumn{2}{|c|}{ Performance of the stations } \\
\hline & 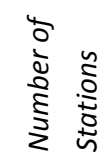 & 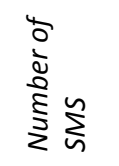 & 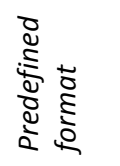 & 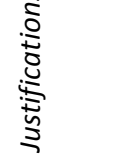 & $\begin{array}{l}\frac{5}{5} \\
\text { जิ } \\
\text { जे }\end{array}$ & 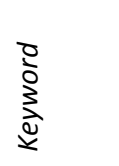 & 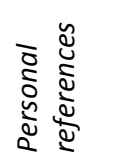 & 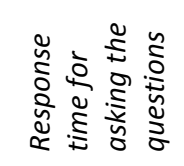 & 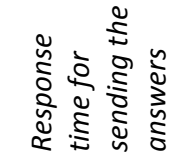 \\
\hline $\begin{array}{l}\text { Round } 1 \\
\text { baseline }\end{array}$ & 9 & 515 & 30.6 & 18.0 & 61.9 & 75.3 & 59.3 & 16.3 days & 7.1 days \\
\hline Round 2 & 9 & 526 & $40.3^{* *}$ & 16.5 & $24.7^{* * *}$ & $93.8 * * *$ & 53.5 & 20.1 days & 6.6 days \\
\hline Round 3 & 3 & 191 & $64.4^{* * *}$ & 13.3 & $11.8^{* * *}$ & $83.3^{*}$ & $31.2 * * *$ & 13.3 days & 1.7 days \\
\hline Round 4 & 7 & 607 & $80.9 * * *$ & $89.9 * * *$ & $17.8^{* * *}$ & $80.9 *$ & $70.0 * * *$ & 6.3 days & 2.6 days \\
\hline Round 5 & 5 & 579 & 30.1 & $67.0 * * *$ & $21.3^{* * *}$ & $61.4^{* * *}$ & $84.6 * * *$ & 1.75 day & 2.5 days \\
\hline Round 6 & 3 & 357 & $85.3^{* * *}$ & $86.7^{* * *}$ & $12.1 * * *$ & $87.3^{* * *}$ & $75.5 * * *$ & 2.3 days & 5.8 days \\
\hline
\end{tabular}

Note: ${ }^{*} p<.05 ;{ }^{*} p<.01,{ }^{* *} p<.005$ (z-test for comparing the round proportion with the baseline proportion in that variable)

The number of stations involved in each round was inconsistent across the quasi-experiment due to external factors that the CGHR team had little control over, such as Africa's Voices representative(s) permanently or partially leaving the station (Buddu FM in Uganda and Chipata, Zambia); permanent failure in the SMS technology (Gbafth in Sierra Leone); changes in the management structure of the station (Mudzi Wathu in Malawi); and challenges in communicating with the stations (Radio Ulanga in Tanzania and Rite FM in Ghana). These hindrances to the research process would have been overcome in a context with fewer human, financial and time 
constraints, but due to the limited funding and capacity of the CGHR team, it was not possible to offer viable solutions to these stations. This was particularly reflected in the last rounds (5 and 6 ) due to a cumulative "station attrition" effect.

Type of question. The insights from the pre-pilot question revealed that when listeners were faced with four multiple-choice options, the level of participation was low (17 SMS received in total), the SMS were not received in the predefined format (e.g., missing keyword and/or pre-defined option) and no explanations were given. When only two options were offered (rounds 1, 2, 4, 5 and 6), the participation increased considerably, as more SMS were received from audiences, and richer answers were spontaneously sent, potentially signalling that listeners engaged with the topic to a greater extent.

When general options such as yes/no were presented (rounds 1 and 6), the justifications in the messages were not always consistent with the options chosen. For example, the predefined answer "BAG NO" which means "I do not agree with our government banning the use of plastic bags" was sometimes followed by incoherent justifications, such as "because plastic bags are harmful for the environment" (listener from RiteFM in Ghana). This problem may be related to listeners' experience of memorizing how the answer's options relate to the question as well as the use of a mental association of the keyword linked to the answer (for example, "BAGS NO" meaning wrongly "I don't want bags"). Audiences can be easily misguided by previous messages read on air that were wrongly phrased, as SMS with inconsistent answers seem to appear in a temporal sequence, and are more common in some stations in particular rounds. Questions with two short and clear option alternatives (e.g., AIDS vs. Malaria in round 5) are not prone to these inconsistent answers. Thus the binary question seems to be the most appropriate type for pollilliterate populations as they were better understood and seemed to provoke more automatic answers. Importantly, the evidence base for adjusting and tailoring question formats to audience specificities comes from open-format question styles, asking "why?". Close-answer formats, by contrast, are liable to generate false confidence in understanding audience comprehension and intent.

Format of answers. Across all the stations, respondents showed a clear preference for sending longer SMS containing arguments supporting the answer and personal information rather than predefined closed messages. In rounds 1 to 3, the instructions contained in the script of the question sought SMS in a standardized format (keyword+closed answer) but some SMS contained justifications supporting the options chosen (e.g., "Bags No-they help us to carry things in an easy and fast way but where to dump them we have to take care"- BreezeFM in Zambia).

In rounds 4 to 6 , a 'why question' was asked to prompt the provision of arguments/reasons. As expected, the majority of messages (65-90\%) contained justifications of the answers. This result suggests that listeners are often eager to send explanations justifying their choice when responding to a question, and that most feel motivated or compelled to do so when prompted. For example, in round $5,89.9 \%$ of SMS contained justifications compared to less than $20 \%$ in rounds 1 to 3 where no justification was sought. The explanations are useful to contextualize crude percentages and, in some cases, they suggest that the question was not well understood or it was answered erroneously. Thus insights into comprehension, implementation error and determinants of participation are revealed through long-form SMS answers in informative and instructive ways.

Listeners sometimes use the mediated public space of Africa's Voices as a channel to communicate with the stations and other listeners or to explicitly seek visibility in their community. As such, some messages also include public announcements, greeting to presenters, friends and family, 
offers of services or jobs and feedback about the show or the quality of the transmission. Thus the motivations for participation are multiple and diverse in nature, and a methodology that anticipates and accommodates this, despite drawbacks for the analyst, has unique merits.

Audiences from different stations seem to have different preferences on modes of communication. For example, in some stations, listeners tend to call in to answer the question on air, while in other stations, respondents send only SMS. When reflecting on preferences for voice vs. textual communication, the characteristics of the population and the costs of calls and SMS need to be taken into consideration. In the absence of socio-demographical information about the participants in Africa's Voices, one obvious insight from the pilot is that in more rural stations (e.g., Radio Ulanga in Tanzania) audiences prefer communicating by voice.

This evidence creates a tension between whether Africa's Voices should be built around existing modes of communication or whether it should be more active in introducing and encouraging new ways of communication between radio stations and audiences. On the one hand, however limiting the modes used may limit the free expression of opinions and may create artificial ways of communication, on the other hand, standardized short messages help to ensure comparability of answers in different languages and countries.

Language and wording of questions. Audiences tend to answer in the language in which the question was asked. In the first round, the question was asked in English and the majority of SMS were also in English (62\%). In subsequent rounds, the questions were asked in local languages and over $75 \%$ of SMS were also written in local languages (e.g., Swahili, Chichewa/Nyanja, Luganda, Luo and Krio) although some messages were in English. Drawing on insights from earlier rounds, the Africa's Voices team embraced natural forms of expression and sought ways to encourage a diverse range of listeners to participate through SMS written in any language, whether English or local.

The increased focus of Africa's Voices on collecting opinions in the context in which they were generated and in their natural forms of expression not only promotes inclusivity but also, importantly, enhances the authenticity of what is communicated, due to stronger linkages to audience's actual practices, self-reflections and social identities. This clearly raises different translation-specific issues, and creates methodological and resource challenges. So far, the Africa's Voices team is in charge of translations into English from local languages. However, back translation procedures would be desirable to check the accuracy and comparability of the translations carried out independently. This procedure had not yet been put into practice due to lack of resources during the piloting stage. There are also opportunities to innovate with semantic and linguistic analysis approaches and adapted 'data scraping' tools.

Use of keywords. When comparing the concrete keywords (e.g. BAG, FEAR, MARRY, SAFE) with abstract but short keywords (e.g. AV, AA), surprisingly, abstract keywords seem to be used more ( $94 \%$ in rounds 2 and $83 \%$ in round 3 vs.61 to $87 \%$ in rounds 1 , and 4 to 6 ). Because the messages without keywords cannot be filtered automatically, an additional effort to identify and export messages is required from the stations. In this process, some of the messages can get lost, especially in stations that received a high volume of SMS. Thus, these percentages may not accurately represent the percentage of audiences that use the keyword (the percentages are probably lower).

More importantly than the type of keyword (concrete vs. abstract), the way that the keyword is understood affects the content of the answer. When a concrete keyword is used, it must be related to both the content of the question and of the answer. For example, in round 1, the 
keyword BAG distorted the meaning of the answers yes/no, as already mentioned. In round 6 , the keyword SAFE, was more successful because it relates to the content of the question and of the answer. For example, the answer "SAFE yes" to the question "Do you feel safe to walk in your area after dark?" conveys only one possible and clear meaning ("I feel safe").

Anonymity request. A clear finding from the Africa's Voices pilot is that despite the efforts to encourage anonymity, most respondents prefer to identify themselves in the messages. In all radio stations, selected messages are read aloud in the airwaves because the presenters believe that this will boost audience participation and the popularity of the programmes. The correlate of voice is recognition, and the significance and attraction of publicity within a stimulated debate/discussion forum is vital to the programme's ability to succeed. As noted by the Head of Programmes from Radio BreezeFM in Zambia:

No other topic was done apart from reading the scripts and reading the incoming messages. Our listeners cannot send messages if you don't read what they send. Even when you don't read their names, they stop sending. So keeping them unknown stops them from sending messages." (Martin Mwape, Radio BreezeFM)

The Africa's Voices research team nevertheless has sought to navigate these dynamics in order to maintain ethical standards. The appeal for anonymous messages was made with different levels of insistence through the pilot rounds and with differential impact. Unsurprisingly, weak anonymity instructions produced more messages with personal information (e.g., $84.6 \%$ in round 5). Recognition of one's opinion is linked with individuality. A statistically significant association was observed between sending arguments supporting the answer and sending personal information, $(x 2(1)=18.18, p<.001)$ reinforcing the idea that listeners send personal information to seek recognition in the community for their opinions. However, rather than being a thoughtless generalized practice, audiences seem aware of anonymity threats as they opt for not sending personal clues when providing more sensitive information. For example in round 4 , messages with the answer "Fear AIDS" showed significantly lower personal references than messages with answers of "Fear Malaria" ( $\chi 2(1)=51.19, p<.001)$.

Assuming that listeners may send personal information in the messages, the impact of social desirability in the answers needs careful consideration in relation to the selection of topics and designing of questions. The tendency to answer in socially desirable ways to project a positive image of the self may be embedded in the cultural milieu and linked to perceived social norms. Particular words and expressions can also be more prone to social desirability in particular languages or cultures. The effect of social desirability can be minimized by pre-testing the wording of the question and the script that introduce the question, using for example cognitive interviewing methods. By elaborating arguments of the two sides of the topic and seeking contrasting opinions, Africa's Voices scripts prompt more authentic opinions. The presenters were also instructed not to take a stance on the topic, to welcome all opinions and to create a genuine debate by balancing the number of contrasting answers that they read aloud. This procedure enriches and encourages debate and signals to listeners that they may express freely their opinions in a non-judgmental context.

Engagement of the stations. The radio stations involved in the pilot varied considerably in their capacity and engagement with Africa's Voices. Some stations were actively involved in all stages of the project, suggesting topics, contributing to the design of questions and scripts, sending feedback about audience's participation and impact of the project in the communities, exporting messages promptly and discussing the results with the Africa's Voices team. As a rule, stations with lower technological capacity, irregular patterns of communication and more bureaucratic 
approaches were more passive in their contribution to the development of project. In the feedback questionnaire that was sent to the presenters after round 1, slow internet connections and software failure were identified as the main challenges that these stations faced during the pilot. These issues seem to have had a bigger impact in the first rounds provoking delays in the implementation of the project in particular stations. However, in all the stations, as the presenters became familiar with the software and the Africa's Voices procedure, their performance in the pilot demonstrably improved. On average, the response time between receiving the final question, asking the question and sending the results has dropped from 23 days in round 2 to 9 days in round 4 and 4 days in round 5 .

When exposed to new software and an innovative model for interactive shows, stations needed time to learn and integrate them into existing habits and practices. After the first six months of the pilot, some radio stations had created new programmes to accommodate Africa's Voices questions. These weekly programmes were devoted to discussing local and national issues, adopting the same format as Africa's Voices, asking questions to be answered through SMS using FrontlineSMS. The programme 'What's your view' (Inu Mukutanji) in Radio Mudzi Wathu in Malawi or the programme 'People's viewpoint' (Maganizo ya anthu) in BreezeFM in Zambia are examples of this.

Other stations have extended the Africa's Voices model to other programmes. For example, Radio NamLolwe, drawing on the success of Africa's Voices, launched a programme with a focus on labour issues, inviting listeners to answer questions by SMS that are read out and discussed on air. In BudduFM in Uganda, the Africa's Voices programme was adjusted to the station's practice of using vox pop in the communities. The programme 'Our Parliament' was held during an open-air session in the community inviting opinions from citizens to be discussed by local leaders (round 5 on early marriage).

During the pilot, the stations did not regularly engage experts and political leaders in the Africa's Voices debates as they were still experimental in nature and were limited in scope. The presenters seemed motivated however to inform authorities about audience opinions, as expressed by one presenter from Nkhotakota Community Radio in Malawi, when he received the results of plastic bags round:

Thank you for sharing the results with us, this is very important as we know how important it is engaging communities in making decisions and policies. As matter of fact we are going to share the results with relevant authorities as well. (Phillipp Chinkhokwe).

\section{Discussion}

Through the Africa's Voices pilot project, CGHR successfully explored how SMS-based interactive radio formats can create new spaces for voice and discussion with the potential to generate valuable comparative data. With further development and analysis, this data could be aggregated into public opinion indicators linked to the context of local debates and knowledge. However, this unique opportunity to access and analyse voices from hard-to-reach African populations using new ICTs poses another set of pressing analytical, ethical and methodological challenges: the balance between individual uniqueness and aggregation; the compromise between anonymity and recognition; and the interplay between structured technology interfaces and workflows and open spaces for voice and discussion anchored in their social contexts. 


\section{Big Data and Aggregation of Unique Voices}

One challenge that the pilot project presented was how to encourage particular forms of participation that do not lend themselves to easy aggregation and analysis. The desire to allow for individual expressions of opinion whilst seeking to learn at aggregate levels reflects a wider urgent issue for action oriented research seeking to capitalize on Africa's digital communications revolution: how to encourage, value and protect unique voices while seizing the opportunities presented by ease of gathering and aggregating larger volumes of data. The same technological innovations that are enabling new voices to be expressed are also making it possible for them to be aggregated and abstracted with greater ease.

Methodologically, this intersects with a current concern in the social sciences: making use of big datasets characterized by volume, variety and/or velocity (Eaton et al., 2012) that were 'organically' created through new communication technologies (internet and mobile phones). The footprint left by users of these technologies contains the potential to understand human behaviour in real-life contexts at a large scale of cultures and social groups. But the possibilities of using streaming and unstructured data for social research remain underexplored (Gonzalez-Bailon, 2013) mainly because the tools for handling and analysing 'big data' have not yet been adopted by social researchers. Often big data is unstructured, mixed-format and incomplete. However, its usefulness may not directly correspond to its complexity. Additionally, organic data carry imperfections that threaten the validity of social research: self-selection of cases; self-presentation bias; few covariates; and privacy and access issues (Couper, 2013).

Moving away from a survey-type approach (for example, featuring probability sampling, panels of respondents, or closed format questions), Africa's Voices has gradually adopted an approach that produces data that is more rich and dynamic led by the pilot insights about audiences' preferred ways of participation in public discussions through interactive radio and SMS. This approach resulted in large volumes of texts in multiple languages and mixed formats (textual and predefined answers) whose meaning cannot be detached from local realities. Yet this type of data can capture, in a genuine and meaningful way, public opinion in hard-to-reach communities, at the expense of conventional scientific canons.

The usefulness of such data to capture cross-country variations, given by the direct comparison of simple percentages and themes, is limited by the non-representativeness of samples, which may lead to flawed interpretations. Such samples are however adequate to capture the uniqueness and contextual embeddedness of voices, the richness of arguments and the dynamics of discussion and the formation of opinions.

The future for Africa's Voices is thus to move in the direction of exploring innovative tools for analysing big data that allow meaning to be extracted from unstructured data in voice and text formats (technology on speech recognition, machine learning, natural language processing) and to provide real time insights based on emerging data (big data streaming).

\section{Anonymity and Recognition}

A central finding of the pilot is that having a voice through the 'publicity' generated by radio matters, as audiences from different radio stations engage in debates on the airwaves either by calling in or sending SMS. Recognition of one's own voice and contribution to the debate (or even the hope of contributing) or the vicarious sense of recognition that comes from a space in which others "like you" participate, seems to be a key factor in participation in Africa's Voices polls. Yet the research team's concern for protecting anonymity in Africa's Voices data generates a tension 
between local recognition and research ethics. To some extent, anonymity does not seem to be a concern for audiences as they are eager to send information that reveals their identity. The question remains to what extent that does, or should, apply.

Despite all the efforts to ensure anonymity of participants at the point of communication (although their mobile phone numbers are unavoidably communicated), people prefer to send their names, residence, and sometimes the occupation and names of family members. If the answers gave more elaborate reasons for their position, it is more likely that they will be accompanied by personal details, supporting the idea that audiences seek recognition for their opinion.

The opportunity to express their opinions and the actual or anticipated acknowledgement of taking part in a relevant discussion by other members of the community seem to be significant drivers for audience participation in Africa's Voices programmes. The public character of the discussion and the accessible format are designed to invite participation from different social groups. However, a deeper study of the characteristics of the individuals who participate in Africa's Voices in one radio station in Kenya (Radio NamLolwe) showed a marked gender imbalance with about two thirds of the messages sent from male listeners. Some bias towards younger ages and occupation (mainly farmers) was also noted but generalizations from this sample to audiences of other radio stations are not obviously possible. However, this ad hoc finding suggests that Africa's Voices debates may reflect existing cleavages in participation by offering an alternative platform for those people who already have a voice that is acknowledged by the community. This trend, and exceptions to it, requires further exploration.

A complete picture of participation would require a more comprehensive study where sociodemographic information of the participants is collected on a continuing basis. The dynamics of participation could thus be analysed over time in order to understand whether these debates create new and more inclusive forms of participation, or if they only contribute to reinforce existing bias. This next stage of the project might incorporate innovations in communications software tools that enable interactive sessions with participants are automatically triggered at the time of initial participation, and that link individual voices to with socio-demographical profiles, gathered in real time.

\section{Open Platforms for Voice and Discussion}

Identifying the conditions that encourage and sustain expression of voice and debate helps to understand the nature of participation in Africa's Voices. Valuing voice implies encouraging individual opinion and narratives and creating spaces where these voices can be heard. It also suggests that participants perceive that voice matters for making a difference to improve their lives and the lives of others. The outcomes of these practices require further publicity and engagement with relevant decision-makers and institutions to promote their usefulness and thus sustainability. Process-tracing the instrumental usefulness of such participatory practices is evidently challenging, but anecdotal analysis can and should be pursued to help form a credible picture. Some successful examples publicized by the stations can help to strengthen audience perceptions that expressing voice really can matter, for it is crucial that audiences can grasp exactly how their voices could reach relevant authorities and be taken into account for decisionmaking. A detailed examination of such processes forms the core of related CGHR research projects, but this dimension of Africa's Voices was only superficially considered during the pilot stage. 


\section{Conclusion}

The pilot of Africa's Voices explored how new communication technologies can be combined with traditional media to enable public spaces for discussion and debate about topics relevant to all audiences. At the same time, the opinions of hard-to-reach populations can be organically gathered, using free software, and aggregated into indicators of public opinion linked to social contexts and knowledge. The pilot collected the views of communities in East, Central, West and Southern Africa, underscoring the potential for a continental dialogue, in multiple languages, on common topics that concern all of the communities involved.

The format and content of radio-mediated communications within programmes that permit open formats might not differ as dramatically as we might assume from spontaneous conversations as they occur in other public spaces. Audiences tend to express their opinions in local languages, combining standardized answers, required for simple aggregation and comparability, with reasons justifying their choices.

The methodological decisions, guided by the feedback from audiences and radio stations, shaped Africa's Voices as a viable platform for public opinion gathering. As an innovation valuing individual voice, spontaneously expressed in inclusive public spaces, and immersed in everyday real-life contexts, Africa's Voices distances itself from classical methods of gathering public opinion such as surveys or focus groups. The scalability and sustainability of such a platform, however, depends on a sharper focus on what value is generated, where in the process and for whom, as well as how knowledge assets can be developed, and how it can be tied to possible funding/revenue and partnership models.

Africa's Voices is built around three tensions: the balance between individual uniqueness and aggregation; the compromise between anonymity and recognition; and the interplay between structured technology interfaces and workflows, and open spaces for voice and discussion anchored in their social contexts. The future of Africa's Voices will capitalize on opportunities created by responding innovatively to these tensions, exploring tools for aggregating and analyzing 'messy' data; technological solutions for gathering socio-demographical information from audiences; and engagement strategies for political and development actors. Its aim will be to maximize the impact of expanding and enriching the voice of African citizens in the public square. 


\section{$\underline{\text { References }}$}

Arendt, H. (1963). On revolution. London, Faber and Faber.

Arendt, H. (1971). The human condition. Chicago, University of Chicago Press.

Bertot, J. C., Jaeger, P. T., \& Grimes, J. M. (2010). Using ICTs to create a culture of transparency: EGovernment and social media as openness and anti-corruption tools for societies. Government Information Quarterly, 27(3), 264-71.

Bratton M., Mattes, R. \& Gyimah-Boadi, E. (2005). Public opinion, democracy, and market reform in Africa. Cambridge University Press.

Brisset-Foucault F. (2009). Polis Academy: Talk shows radiophoniques, pluralisme et citoyenneté en Ouganda, Politique Africaine, 113, 167-186.

Chiumbu, S. H. (2013). Communities of strangerhoods?: Internet, mobile phones and the changing nature of radio cultures in South Africa. Telematics and Informatics, 30(3), 242-251.

Cook, T. D., \& Campbell, D. T. (1979). Quase-experimentation: Design and analysis issues for field settings. Boston, MA: Houghton Mifflin Company.

Couldry, N. (2010). Why voice matters: Culture and politics after neo-liberalism. London: SAGE.

Couper, M. (July 2013). Is the sky falling? New technology, changing media and the future of surveys. Keynote presented at the fifth conference of the European Survey Research Association.

Eaton, C., Deroos, D., Deutsch, T., Lapis, G., \& Zikopoulos, P. (2012). Understanding big data. London: McGraw Hill.

Fishkin, J. (1991). Democracy and deliberation: New directions for democratic reform. Cambridge University Press.

Fishkin, J. (2009). When the people speak: Deliberative democracy and public consultation. Oxford University Press.

Gagliardone, I. (2009). The socialization of ICTs in Ethiopia: Reshaping technology for nation building, Journal of Socio Technology and Knowledge Development 1(4): 51-67

Gratz, T. (2013). New media entrepreneurs and changing styles of public communication in Africa: Introduction. Journal of African Cultural Studies, 25(1), 1-13.

Gonzalez-Bailon, S. (2013). Social science in the era of big data. Policy and Internet, 5(2), 147-160.

Habermas, J. (1991). The structural transformation of the public sphere: An inquiry into a category of bourgeois society. Boston: MIT Press.

Heeks, R. (2008). ICT4D 2.0: The Next Phase of Applying ICT for International Development, Computer, 41(6):26-33.

Kleine, D. (2010). ICT4WHAT?-Using the choice framework to operationalise the capability approach to development, Journal of International Development, 22: 674-692.

Mare, A. (2013). New media, pirate radio and the creative appropriation of technology in Zimbabwe: Case of Radio Voice of the People. Journal of African Cultural Studies, 25(1), 30-41.

McIntosh, J., (2010). Mobile phones and Mipoho's prophecy: The powers and dangers of flying language. American Ethnologist 37, 337-353.

Morawczynski, O. (2009). Exploring the usage and impact of "transformational" mobile financial services: the case of M-PESA in Kenya, Journal of Eastern African Studies 3(3): 509-525. 
Moyo, L. (2013). The digital turn in radio: A critique of institutional and organizational modeling of new radio practices and cultures. Telematics and Informatics, 30(3), 214-222

Moyo, L. (2013). Introduction: Critical reflections on technological convergence on radio and the emerging digital cultures and practices. Telematics and Informatics, 30(3), 211-213.

Moscovici, S (1961). La psychanalyse, son image et son public. Paris: Presse Universitaires de France.

Myers M. (2008). Radio and development in Africa: A Concept paper, IDRC. http://www.dfid. gov.uk/r4d/PDF/Outputs/ICT4D/Radio_and_Development_in_Africa_concept_paper.pdf.

Nyamnjoh, F. (2005). Africa's Media: Democracy and the Politics of Belonging. London: Zed Books.

Odugbemi S. \& T. Lee eds. (2011). Accountability through public opinion: From inertia to public action. Washington: World Bank Publications.

Schulz D. E. (1999). In pursuit of publicity: Talk radio and the imagination of a moral public in urban Mali. Afrika Spectrum, 34(2), 161-185.

Shadish, W. R., Cook, T. D., \& Campbell, D. T. (2002). Experimental and quasi-experimental designs for generalized causal inference. Boston: Houghton-Mifflin.

SIDA (2009). ICTs for Democracy: Information and communication technologies for the enhancement of democracy - with a focus on empowerment. SIDA and Association for Progressive Communications.

Spitulnik D. (2002). Mobile machines and fluid audiences: Rethinking reception through Zambian radio culture. In Ginsburg, F., Abu-Lughod, L. \& Larkin, B. (Eds.), Media worlds: Anthropology on new terrain (pp 337-354). Berkeley and Los Angeles: University of California Press.

Sen, A. (1999). Development as Freedom. Oxford University Press.

Srinivasan, S. (2007). No democracy without justice: Political freedom in Amartya Sen's capability approach. Journal of Human Development, 8(3), 457-480.

Srinivasan, S. (2014). FrontlineSMS, Mobile-for-Development and the 'long tail' of governance. In Livingston, S. \& Walter-Drop, G., Bits and atoms: Information and communication technology in areas of limited statehood. Oxford University Press.

Sudman, S., Bradburn, N. \& Schwarz, N. (1996). Thinking about questions: The application of cognitive processes to survey methodology. San Francisco: Jossey-Bass Publishers.

Tajfel, H. \& Turner, J.C. (1986). The social identity theory of intergroup behaviour. In S. Worchel and W. G. Austin (Eds), Psychology of Intergroup Relations (pp 7-24). Chicago, IL: Nelson-Hall.

Vokes R. (2007). Charisma, creativity and cosmopolitanism: a perspective on the power of the new radio broadcasting in Uganda and Rwanda. Journal of the Royal Anthropological Institute, 13(4), 805-824.

Wasserman, H. (2011). Mobile Phones, Popular Media, and Everyday African Democracy: Transmissions and Transgressions Popular Communication 9(2): 146-158.

Willems, W. (2013). Participation-In What? Radio, Convergence and the Corporate Logic of Audience Input Through New Media in Zambia, Telematics and Informatics, 30(3): 223-231.

Wolf, T. (2009). Poll poison?: Politicians and polling in the 2007 Kenya election. Journal of Contemporary African Studies, 27(3), 279-304. 
Centre of Governance \& Human Rights

Citation: Abreu Lopes, C., Srinivasan, S., (May 2014) 'Africa's Voices: Using mobile phones and radio to foster mediated public discussion and to gather public opinions in Africa', CGHR Working Paper 9, Cambridge: University of Cambridge Centre of Governance and Human Rights

You are free:

to copy, distribute, display, and perform the work

to make derivative works

Under the following conditions:

Attribution - You must give the original author credit.

Non-Commercial - You may not use this work for commercial purposes.

Share Alike - If you alter, transform, or build upon this work, you may distribute the resulting work only under a licence identical to this one.

Please see full details of this license here: http://creativecommons.org/licenses/by-nc-sa/2.0/uk/ 